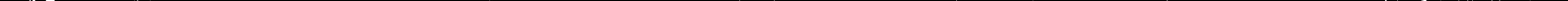




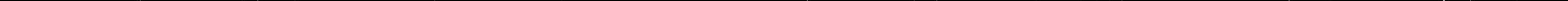


LBL-33235

UC-410

LSGN-119

\title{
SPECTRAL QUALITY OF ALS U5.0 UNDULATOR AND FIELD ERROR EFFECTS
}

\author{
C. WANG, S. MARKS, and B. KINCAID \\ ACCELERATOR and FUSION RESEARCH DIVISION \\ Lawrence Berkeley Laboratory \\ University of California \\ Berkeley, CA 94720
}

MAY 1993

This work was supported by the Director, Office of Energy Research, Office of Basic Energy Sciences, Materials Sciences Division, of the U.S. Department of Energy under Contract No. DE-AC03-76SF00098. 


\title{
Spectral Quality of ALS U5.0 Undulator and Field Error Effects
}

\author{
C. Wang, S. Marks and B. Kincaid \\ Lawrence Berkeley Laboratory, University of California, Berkeley, California 94720
}

\section{Abstract}

The first insertion device of the Advanced Light Source (ALS), a U5.0 undulator, has been carefully adjusted and qualified with a specially designed magnetic measurement system. The magnetic field of the undulator has been fully mapped at a series of gaps with very high accuracy. Based upon these measured field data, we evaluate the radiation spectral quality of this device in terms of an ideal sinusoidal device and examine the field error effects. Moreover, Statistical correlation between the field errors and radiation degradation is examined by using the large quantity of magnetic field data sets accumulated in the process of adjusting and qualifying the U5.0 undulator.

\section{INTRODUCTION}

In order to obtain high brightness photon sources, a low emittance storage ring and long insertion devices have been implemented at the ALS. However, it is well known that the magnet field errors tend to degrade the performance of an insertion device. To achieve the high performance required, state of the art technology is employed to build the insertion devices and the magnetic measurement system. The first insertion device being installed at ALS is the U5.0 undulator [1]. Before installation, it was carefully adjusted and qualified based upon magnetic measurements [2] as well as radiation calculations using the measured field data. In this report, we present some results that demonstrate the quality of the US.0 radiation spectrum. Instead of showing the general performance of U5.0, which is available in earlier publications [3], we pick up a few representative cases and present a detailed spectral comparison between an ideal sinusoidal field and the measured real device.

In the process of adjusting and qualifying the U5.0 undulator, a large number of magnetic field data sets have been accumulated. This collection of data consists of a statistical assembly that represents real devices with different field errors. It is interesting to examine the correlation between spectral quality and field errors. This is the first time that such a real measured data set assembly is available. We will show the statistical correlation between the field errors and radiation degradation and compare it with earlier computer simulation studies. [4]

To examine the magnetic field error effects on spectral quality, all spectrum properties are calculated using the measured field data, and then normalized by the values calculated using ideal field, which consists of a sinusoidal field and one half peak pole at each end. The radiation spectral calculations are done with program RADID, whose undulator radiation calculation algorithm is based on Ref. 5. The magnetic field analysis are done with a program ANALYZE [6].

\section{SPECTRAL QUALITY OF U5.0 UNDULATOR}

The U5.0 undulator has 89 periods of $5 \mathrm{~cm}$ each. It is designed to produce high brightness radiation from $50 \mathrm{eV}$ to $1.9 \mathrm{keV}$ [3] by using up to the 5 th harmonic. From B. Kincaid's theory [7] about random field error effects in undulators we know that, in the small error limit, for an $\mathrm{N}$ period undulator with relative rms random field error $\sigma$, the nth harmonic peak flux density is degraded by a factor of $e^{-30 q}$, where

$$
q=n^{2} \sigma^{2} N\left[\frac{\frac{K^{2}}{2}}{1+\frac{K^{2}}{2}}\right]^{2}
$$

Since the factor decreases exponentially with $n^{2} \mathrm{~N}$ for a given $\sigma$, it applies a stringent requirement on the magnetic field errors in order to keep the peak flux density decrease within $30 \%$ at the 5 th harmonic for such a long undulator. The field errors specification for U5.0 is $\sigma<0.25 \%$.

To accomplish this, special effort was put into design and construction of the device [1]. A specially designed magnetic measurement system was used to adjust and qualify the device after its assembly. Full maps of the magnetic field at a series gaps and off-axis positions were obtained with measurement accuracy of 0.5 Gauss [2]. Such field measurement allows us to examine the quality of the device in terms of spectral performance and storage ring requirements. Here we show the spectral quality of U 5.0 by calculating the on-axis flux density, central brightness and flux accepted within a certain solid angle using real measured field data and comparing results with those from an ideal sinusoidal field.

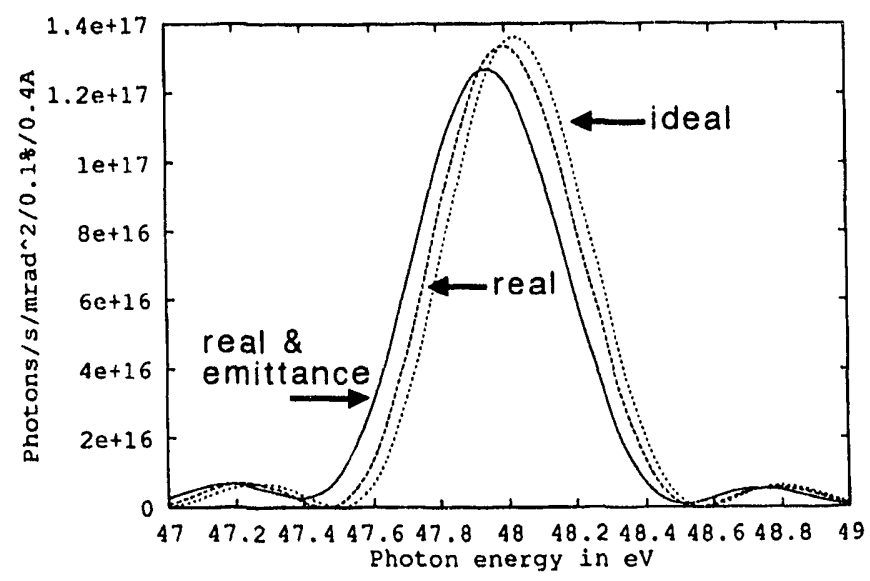

Figure 1. On-axis flux density at $14 \mathrm{~mm}$ gap, 1st harmonic.

Figure 1 shows the on-axis flux density of the first harmonic at the minimum vertical magnet gap, $14 \mathrm{~mm}$. The three curves corresponding respectively to the ideal field, measured 
field and measured field with ALS emittance included. The well known red-shift of peak position and the reduction of peak value are clearly seen, but their effects on spectral quality are negligible. In this case, $\sigma=0.25 \%$. The real spectra are calculated at $69 \mathrm{mrad}$ off axis. A linear least square fit of the trajectory is used to obtain the off-axis angle. The trajectory angle is due to a dipole kick at the end of the device and random electron trajectory walks.

Figure 2 is similar to Figure 1 but shows the 5 th harmonic at a medium gap, $23 \mathrm{~mm}$. The off-axis angle is $18.8 \mu \mathrm{rad}$ in this case. The field error and emittance effects on flux densitv are significant. Though the relative rms field errors is slightly larger $(0.33 \%)$ than the above, the decrease in peak value is much srger due to the higher harmonic number. The emittance effect is also much bigger due to the higher photon energy. However, th eak shape is still quite good and the peak value satisfies the $70 \%$ requirement.

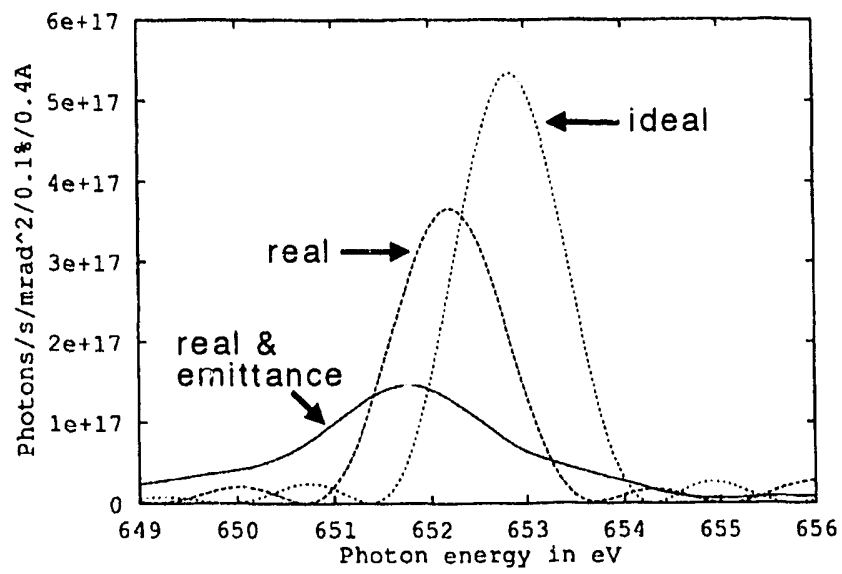

Figure 2. On-axis flux density at $23 \mathrm{~mm}$ gap, 5 th harmonic.

To get a more general picture of the field error effects on the spectral quality, in Table 1, we list the ratios of the real peak flux density to the ideal one for a series of gaps and different harmonics. For reference, the relative rms field errors and the deflection parameter $\mathrm{K}$ at each gap are also listed.

Table 1. Normalized peak flux densities.

\begin{tabular}{|crrrrr|}
\hline & $14 \mathrm{~mm}$ & $18 \mathrm{~mm}$ & $23 \mathrm{~mm}$ & $35 \mathrm{~mm}$ & $47 \mathrm{~mm}$ \\
\hline 1st & $98 \%$ & $95 \%$ & $93 \%$ & $92 \%$ & $93 \%$ \\
3rd & $90 \%$ & $87 \%$ & $85 \%$ & $84 \%$ & $87 \%$ \\
5th & $76 \%$ & $70 \%$ & $69 \%$ & $69 \%$ & $71 \%$ \\
$\sigma$ & $25 \%$ & $0.30 \%$ & $0.33 \%$ & $0.41 \%$ & $0.63 \%$ \\
$\mathrm{~K}$ & 3.974 & 3.008 & 2.132 & 0.973 & 0.453 \\
\hline
\end{tabular}

This table confirms that the spectral quality of this device is quite good at all gaps and meets the design specification. It can be shown that Eq.(1) provides a good prediction of performance up to $23 \mathrm{~mm}$ gap.

It is well known that one figure of merit for a synchrotron radiation source is its central brightness. The field errors may affect brightness in two ways. One is through the degradation of the angular distribution (shape as well as peak value) of single electron flux density. The other is through enlargement of the source size due to random trajectory walks. However, the second one is negligible because the electron beam size is much larger than the amplitude of the single electron orbit, even with the random walks. When considering brightness, electron beam emittance must be taken into account. To evaluate the field error influence on brightness, we calculate the ALS emittance averaged flux densities using the measured field and ideal field. The ratios of corresponding peaks indicate the field error effect on source brightness because the electron beam size effects on both cases are the same. In Table 2 , we list the peak ratios for two typical gaps, $14 \mathrm{~mm}$ and $23 \mathrm{~mm}$

Table 2. Normalized peak brightness.

\begin{tabular}{|cccc|}
\hline & $1 \mathrm{st}$ & $3 \mathrm{rd}$ & 5 th \\
\hline $14 \mathrm{~mm}$ & $98 \%$ & $88 \%$ & $75 \%$ \\
$23 \mathrm{~mm}$ & $91 \%$ & $83 \%$ & $70 \%$ \\
\hline
\end{tabular}

A Monte Carlo simulation is used to take into account the beam emittance. The accuracy of these calculations is about $5 \%$. Comparing Tables 1 and 2 we see that the field error effects on the on-axis flux density and brightness are nearly the same. This is an expected result because the field errors do not change the distribution pattern very much, although the peak value is decreased.

Another figure of merit of a photon source is the flux obtainable in a certain solid angle. Usually, the solid angle for an undulator is the central radiation cone. In Table 3, we show the total flux in a $90 \times 90 \mathrm{mrad}^{2}$ and $180 \times 180 \mathrm{mrad}^{2}$ acceptance angle for the $23 \mathrm{~mm}$ gap case. $90 \mathrm{mrad}$ is about the angular width of the central cone at first harmonic. Because the main effect of field errors is to destroy the constructive interference in an undulator, it has much less effect on the angular integrated flux. The larger the acceptance aperture, the less the field error effect.

Table 3. Normalized flux in different solid angle.

\begin{tabular}{|llll|}
\hline & $1 \mathrm{st}$ & $3 \mathrm{rd}$ & 5 th \\
\hline $90 \mu \mathrm{rad}$ & $39 \%$ & $83 \%$ & $81 \%$ \\
$180 \mu \mathrm{rad}$ & $96 \%$ & $99 \%$ & $92 \%$ \\
\hline
\end{tabular}

\section{CORRELATION OF FIELD ERRORS AND SPECTRAL QUALITY}

In the above section, we have evaluated the spectral quality of the U5 undulator for several on-axis cases which represent the operating regime of the U5. Besides these onaxis cases, many off-axis field scans were also measured in order to characterize the integrated field error distributions. All of these field sets can be viewed as an assembly of real devices with different field errors. These errors are dominated by random errors due to magnetic blocks and manufacturing tolerance. However, there may also be significant systematic errors, especially for off-axis scans. 
Because of the random distribution feature of field errors, only statistical characteristics such as rms value can be used to specify the random field errors. For a specific realization of an error distribution, we can examine its effect on the spectrum as above. However, the general effect of errors on the spectrum can be described only by statistical correlatior, which has been studied analytically by B. Kincaid [5] and with a computer simulation by B. L. Bobbs, et al. [4]. Here we use the measured data set assembly to examine the correlation between field errors and spectral peak flux densities normalized by corresponding ideal values.

Usually, two characteristic values are used to specify field errors. One is the relative rms field error; the other is the rms optical phase error. As pointed out by Bobbs et al. [4], the rms phase error is a better indicator of the device radiation performance.

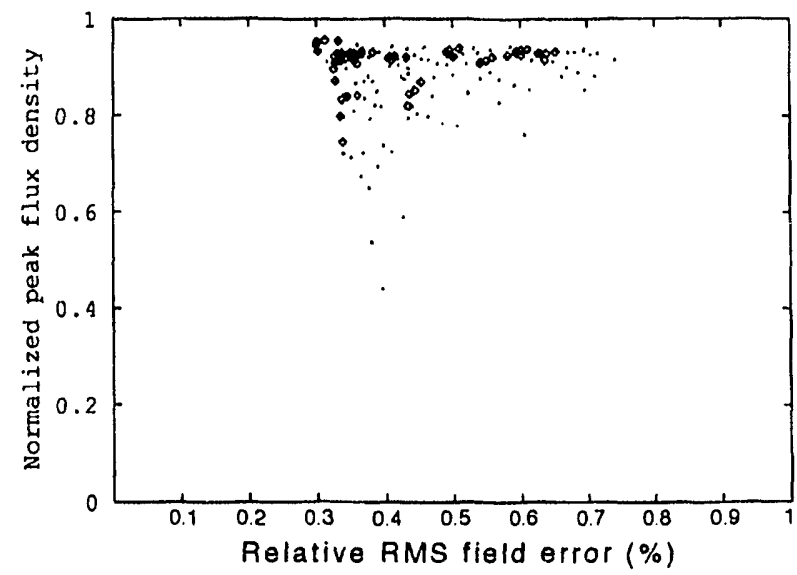

Figure 3. Correlation with relative RMS field errors.

In Figure 3 and 4, we show the correlation between normalized peak flux densities of the first harmonic and the field error characteristic values. Figure 3 uses the relative rms field errors while Figure 4 uses rms phase errors. Each point represents the result for a different field data set. We see that the correlation is not very good for either case. The correlation with phase errors is stronger but surely not as good as the computer simulation result shown in Ref. 4 . In this case, the radiation performance tends to be better than the results from pure random errors. These are probably due to dependency between some data sets and some non random field errors in each set. In fact, if we get rid of the far off-axis cases, we obtain a better correlation as represented by the boxes.

\section{CONCLUSIONS}

The spectral quality of the ALS U5.0 undulator is quite good and satisfies the design requirements of achieving better than $70 \%$ brightness at the 5 th harmonic. The correlations between field error characteristics and spectral performance obtained from different field data set of U5.0 are not as good as former computer simulation results, which assumes random errors only.

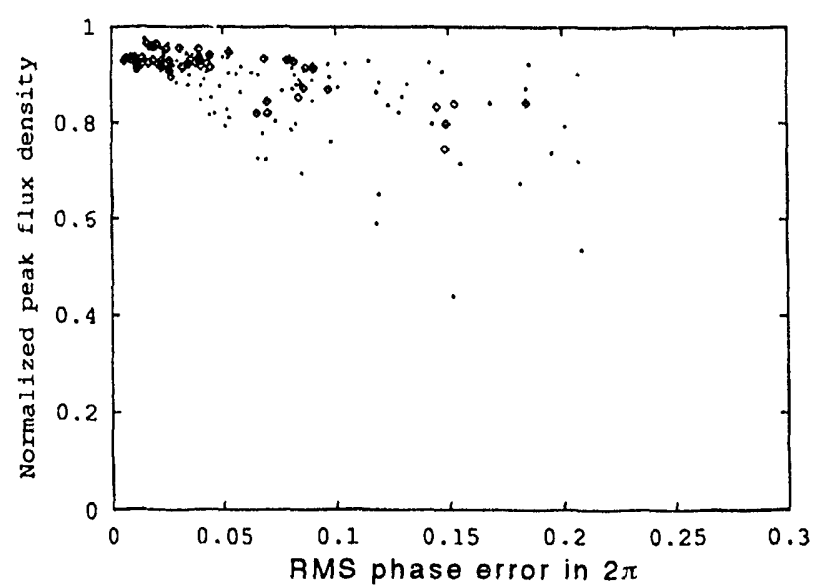

Figure 4. Correlation with RMS phase errors.

\section{ACKNOWLEDGMENT}

This wurk was supported by the Director, office of Energy Research, Office of Basic Energy Science, Materials Science Division of the U.S. Department of Energy, under contract No. DE-AC03-76SF00098.

\section{REFERENCES}

[1] E. Hoyer, J. Akre, J. Chin, B. Gath, D. Humphries,

B. Kincaid, S. Marks, P. Pipersky, D. Plate, G. Portmann, R. Schlueter, W. V. Hassenzahl, "First Undulators for the Advanced Light Source," IEEE PAC (May 1993).

[2] S. Marks, C. Cork, E. Hoyer, D. Humphries, B. Kincaid, D. Plate, A. Robb, R. Schlueter, C. Wang, "Insertion Device Magnet Measurements for the Advanced Light Source," IEEE PAC (May 1993).

[3] "ALS Handbook," Lawrence Berkeley Laboratory Report PUB-643, Rev. 2 (1989).

[4] B. L. Bobbs, G. Rakowsky, P. Kenriedy, R. A. Cover, and D. Slater, "In Search of a Meaningful Field-error Specification for Wiggler," Nucl. Instrum. Methods A296 (1990).

[5] C. Wang, "Concise expression of a classical radiation spectrum," Phys. Rev. E, (June 1993).

[6] J. Bahrdt, B. Kincaid, W. V. Hassenzahl," ANALYZE-a Program for the Analysis of Magnetic Field Data."

[7] B. Kincaid, "Random Errors in Undulators and Their Effects on the Radiation Spectrum," J. Opt. Soc. Am. B/Vol. 2, No. 8 (1985). 

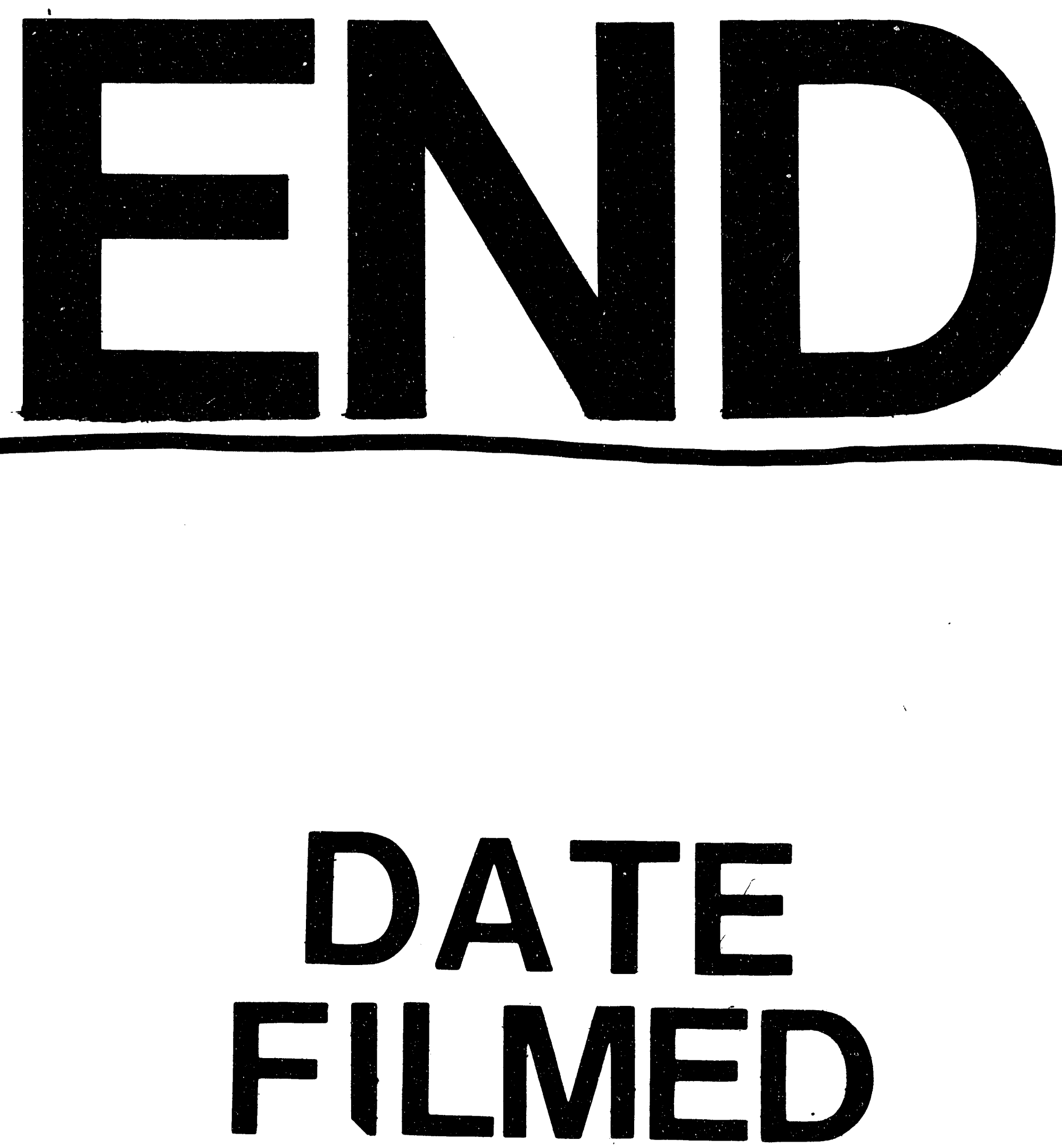

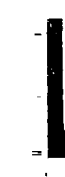

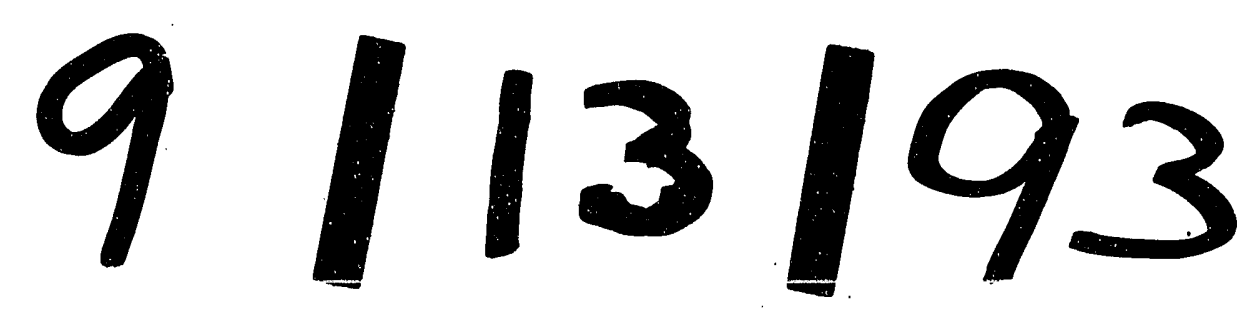


1 